\title{
Robots swarm communication control based on biological behavior inspiration
}

\author{
Mushreq Abdulhussain Shuriji, Traiq Mohammed Salman, Hussein A. Abdulnabi \\ Department of Electrical Engineering, Faculty of Engineering, Mustansiriyah University, Iraq
}

\begin{abstract}
Article Info
ABSTRACT

Article history:

Received Mar 8, 2019

Revised Jun 7, 2019

Accepted Jul 8, 2019

\section{Keywords:}

Biological system

Biomimetic

Robot swarming

Robot swarming, increasingly find importance in the last decade. In these systems, multi mobile robots have to work cooperatively to perform specified tasks. One of the compelling problems is that the robots movements should be in such a way that they should follow a specific guide and at the same time they should have the ability of obstacle avoidance. Inspiriting such movement from biological swarms is a compelling problem. Fish schools, bird flocks and sheep herds are particular examples of biological systems swarming. In this paper, a robot swarming algorithm was developed and proposed based on swarming rules noticed in these biological systems, the combination between the swarm members and the leadership control also explained, a well-design ad-hoc non-essential communication system was proposed for the purpose of use in case of collective takeoff and collective landing swarm-robots, in which activated automatically.
\end{abstract}

Copyright $(2019$ Institute of Advanced Engineering and Science. All rights reserved.

\section{Corresponding Author:}

Mushreq Abdulhussain Shuriji,

Faculty of Engineering, Mustansiriyah University, Baghdad, Iraq

Email: mushreq@uomustansiriyah.edu.iq

\section{INTRODUCTION}

Presently, Multi mobile robot schemes, have capture the fancies of the researches. As well as, expanded progressively in numerous applications such as: industrial, educational and business. Lots of research applications have carried out in this field for example; factory services, astronomical applications, tasks in restricted and dangerous places, warehouse goods transfer, autonomous flying taxi, underwater inspection and detection and security and defense applications.

Systems that are composed of many reactive units that show self-organized behaviors are too complex compared with the single unit system. Such systems are so-called complex systems. The flocking represents one of the examples of the natural self-organized systems, which can be seen in many biological systems [1]. It represents a collective action, which include the movement of many interactive individuals to a common direction. The flock movement as a group depends on the individual behaviors, such as, keeping a minimum distance between the individuals, avoid being alone, avoid obstacles, avoid predators and following prey. This behavior is achieved although every individual might not be aware of the flock orientation [2-4]. The behavior of individuals within the flocks become the focus of attention by many researchers of various branches of science, for example, robotics, biology, physics, and control engineering [5-13]. Studying the mechanical rules that govern these systems has become a reference in modeling many multi mobile robots systems that are depends on the leaderless algorithm. It became possible to design a swarming model using very easy interaction rules that could be effectively designed in autonomous mobile robots. The flock modeling could then introduce beneficial plans for the development of multiple self-controlled mobile robots [14].

The study and analysis of the system takeoff and landing of these swarms groups of the robot shows the needs to prevent mechanical collision and a well design ad-hoc communication system which is separated permanently and independent of the esential communication system between the base stattion and these 
aircrafts. For that reasons, this paper proposed a robots swarm communication control system to overcome these prolems. In addition, another alternative system of communication is also proposed, which is used only in the case of takeoff and landing in these groups of aircraft and is considered short- in short distance (range) outdoor communication.

\section{AUTONOMOUS MOBILE ROBOTS}

Autonomous mobile robots can be highlighted as electromechanical devices that be able to be programmed to reach numerous objectives. They are getting even more importance and accuracy in engineering manufactures as an instrument of transfer, detect, investigation, and human-free assignments related to its efficient and flexible performance. Furthermore, autonomous robots are appropriate on behalf of work in harsh and risky locations, like handling harmful wastes (radio-active) as well as neutralizing glitches in nuclear plants and reactors. Moreover, autonomous flying taxi, securing constructions, sweeping landscape exploring underwater mines extensively, deep oceans travelling and discovering, etc. There is a nonstop essential association between Autonomous mapping and navigation in one side, and the accessibility of data of the nearby environment in the other. In addition, through distance calculations and measurements or even visual pictures captured by the support of functional external sensors, a sufficient amount of information can be stored and processed [15]. A well designed self-controlled mobile robots must be able to handle tasks as follows [16-18]; learning and gain a comprehensive mapping information of its operational nearby location along with obstacle locations to avoid it, consequently navigational decisions will meet a high standard level of accuracy according to the environmental data which collected by the external sensors. Beside, to meet high percentage of precisions for a predefined mission as well as achieving accurate self-controlled directions and arrangements (autonomously) with no human interference. Also, to route and use optimum path. i.e. the robot should use and follow the shortest path to reach a specific predefined aim and maneuver around the obstacles, plus read and collect the sensor information(data) endlessly and use the updated data to adjust and correct its next move and maneuver to adapt limitless variants around it.

Mobile robots design is based on the integration of various fields of science. For example, dealing with locomotion process require the understanding of robot kinematics, dynamics, mechanism and control theories, and solving the problems of localization and navigation require good experience in computer algorithms, artificial intelligence, information theory, and probability theories. A block diagram of control system for an autonomous mobile robot is shown in Figure 1 [19].

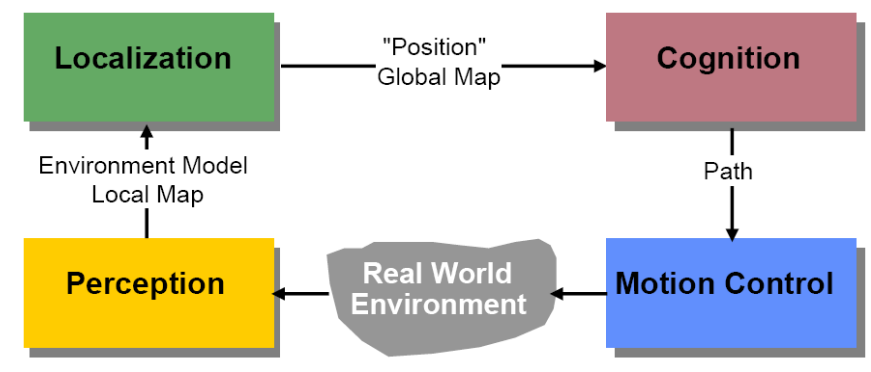

Figure 1. Block diagram of control system for the Mobile Robot [19]

\section{SINGLE ROBOT BASIC MOTION TASKS}

This paper has carried out mobile robots mutual control, besides deliberated a comprehensive review of the basic motions that delivered using a single mobile robot. Bearing in mind a wheeled robot, an extensive literature survey has been accomplished successfully, and found that there are three classifications of a single robot basic motion tasks, which are: point to point, trajectory tracking and path following. The three classifications are shown in Figure 2. 


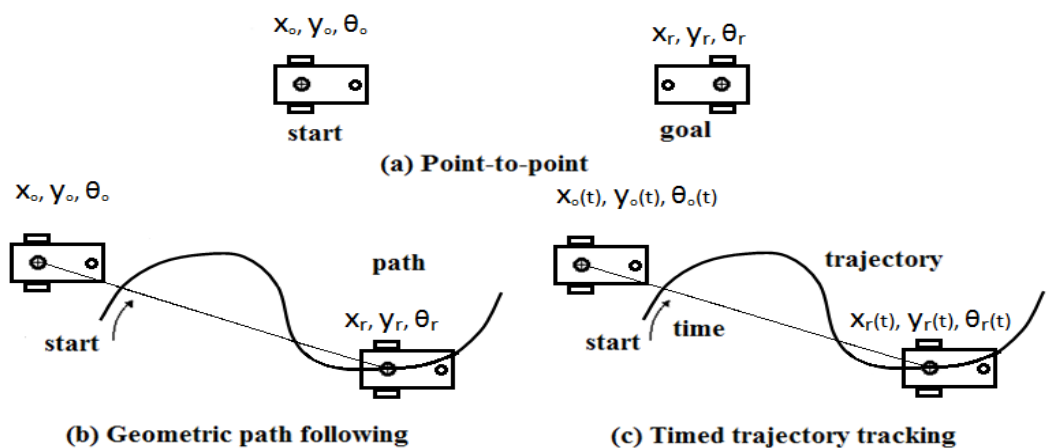

Figure 2. A single robot basic three motion tasks

\subsection{Point-to-Point Motion}

It's also known as a point stabilization. The robot start from known initial configuration all through motion and it have to move to reach roughly required configuration.

\subsection{Trajectory Tracking}

Unlike other type of motion, a robot's reference point have to track a trajectory, which is a geometric pathway with timing associated with the motion, starting from some initial configuration in a Cartesian space. The assigned pathway that determined and delivered from the tracking controller make sure that robot followed it just about time. The tracking controller ensures that the assigned path can be followed asymptotically in time.

\subsection{Path Following}

In this motion, the robot's reference point should track some geometric pathway, without any timing associated with the motion, starting from some initial configuration in a Cartesian space. Regardless, the duration needed to follow and reach the pathway, the controller must guarantee the particular route for the robot to move and stick along with it.

\section{BIOMIMETIC OF SWARMING BEHAVIOR}

The groups of birds and the other biological systems, like herds of sheep, schools of fish, and the likes, move in a harmonic manner. Similarly, ants swarm or birds flock follows other near groups although when they pass in an opposite direction during the seeking for the desired path. The flying of birds individuals within the flock is seems to be identical in either direction and abruptly they might all turn to the right, left or swoop to the bottom toward the ground. It is very astonishing to identify that birds could synchronize and organize their movements precisely. Therefore, to design a model based on flock of birds, a continuous monitoring for a flock of birds is essential to acquire a useful control data. The suggested model is a spatial behavioral one, where bird flying is simulated in a 3D region. Each simulated bird within the flock behaves in an independent manner that is traverses based on its perception for the dynamic environment. Several rules were extracted through monitoring the flock of birds, these rules are;

a. Rule-1: (Collision Avoidance), in this rule, the individuals try to avoid colliding neighboring birds, environment obstacles and borders by keeping a minimum separation with them every times. It has the highest precedence among the rules and it needs continuous observation by animal.

b. Rule-2: (Velocity Alignment), in this rule, the bird try to match its velocity with that of the neighboring birds. If the bird is not executing rule-1, it tries to avoid being isolated from the group by performing attraction behavior towards the group.

c. Rule-3: (flock Centering), in this rule, the bird is trying to fly near neighboring birds to avoid being alone and giving the flock the required cohesion. During that the bird tries to fly toward the center of the neighboring birds.

In the 3D simulation of the three rules above, the variable $\mathrm{N}$ would be used to represents the number of birds, $i$ represents the sequence of the individual where, $(i=1,2, \ldots, N)$, pi is the vector that represents the position of the ith individual, vi represents the unit vector of direction of the ith individual. The simulation time $t$ was segmented at regular step $\mathrm{T}=0.1 \mathrm{~s}$, which corresponds to bird's response time. At each time step, the birds evaluate the position and direction of the neighboring birds in the three non-overlapping behavioral 
regions shown in Figure 3. This information are used to estimate the desired direction for each bird in the next time step di $(\mathrm{t}+\mathrm{T})$ according to specific rules described in the following explanation.

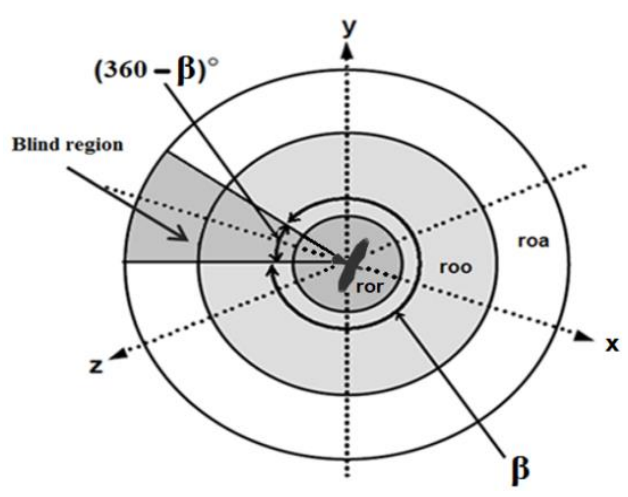

Figure 3. Bird model representation, showing the bird at the center of model regions. $\beta$ : is the field of perception, (360- $\beta)$ : is the blind region behind the bird

Within the region of repulsion (ror), each bird tries to keep a minimum separation distance to other birds. This region was modeled as a sphere of radius rr, and its center lies at the bird position. If the number of neighboring birds that exist within the ror at time $t$ is $\mathrm{nr}$, then the ith bird will respond by flying away and maneuver beyond its neighbors to avoid any possible impact within a particular area, see Figure 4a.

$$
\mathbf{d}_{r}(t+\mathrm{T})=-\sum_{j \neq i}^{n_{r}} \frac{\mathbf{r}_{i j}(t)}{\left|\mathbf{r}_{i j}(t)\right|}
$$

The direction of the unit vector is represented by rij=(pj-pi)/|(pj-pi)| in the direction of the jth neighbor. As we point out above, the specific rule behavior has an extraordinary level of priority as compared with other rules within the model, therefore if $\mathrm{nr}$ not equal to 0 , then next direction of the bird would be $\operatorname{di}(\mathrm{t}+\mathrm{T})=\mathrm{dr}(\mathrm{t}+\mathrm{T})$. The region of repulsion represents the bird's personal space that ensures collision avoidance. If there is no neighbors inside the region of repulsion, i.e. $\mathrm{nr}=0$, then the bird would responds to other birds in the region of orientation (roo) and the region of attraction (roa). These regions have a spherical shape excluding a small blind region lies behind the bird, where it cannot detect other birds when they are there. The blind region, shown in Figure 3 above, is represented by a cone with angle of $(360-\beta)^{\circ}$, and $\beta$ represents the perception field, shown in the same figure.

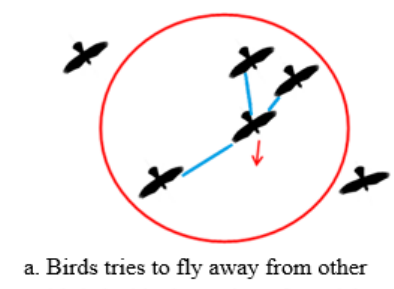

birds inside the region of repulsion.

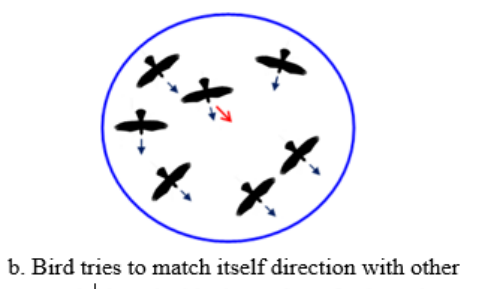

neighbors inside the region of orientation.

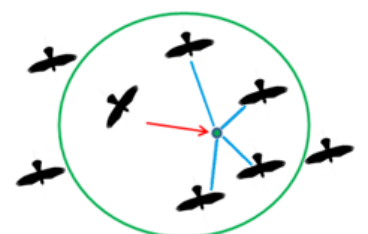

c. The bird is paying attention to be in the center location of its neighbor members within a particular zone of desirability.

Figure 4. various model regions of birds interactions 
The zone of alignment encloses no visible fellow birds apiece using $\mathrm{rr} \leq|(\mathrm{pj}-\mathrm{pi})|<$ ro also, the zone of attraction encloses na visible neighbors apiece by means of ro $\leq \mid(\mathrm{pj}$-pi) $\mid \leq \mathrm{ra}$. A bird will always attempt to rearrange and also aligned with neighbors that surrounded by the region of positioning, and the unit vector of its direction would be calculated using the following equation:

$$
\mathbf{d}_{o}(t+\mathrm{T})=\sum_{j=1}^{n_{o}} \frac{\mathbf{v}_{j}(t)}{\left|\mathbf{v}_{j}(t)\right|}
$$

Realize the Figure 4b, and headed to the center of its surrounding fellow birds in the district of desirability:

$$
\mathbf{d}_{a}(t+\mathrm{T})=\sum_{j \neq i}^{n_{a}} \frac{\mathbf{r}_{i j}(t)}{\left|\mathbf{r}_{i j}(t)\right|}
$$

See Figure 4c. The desirability or attraction is an aim of animals to get together and catchup on with a group, in addition to dodge and not to be lonely. However, the positioning permits a mutual motion by decreasing any possibilities for crashes that may take place among individuals. If the neighboring individuals located only in the row, where $\mathrm{n}=$ no, formerly di $(\mathrm{t}+\mathrm{T})=\mathrm{do}(\mathrm{t}+\mathrm{T})$; similarly if the total neighboring individuals located in the row, where $n=n a$, then di $(t+T)=d a(t+T)$. Equally, if the neighboring individuals located for the two regions, at that point di $(t+T)=1 / 2 d o(t+T)+1 / 2 d a(t+T)$. On behalf of the situations when community powers produces no vector (zero-vector), else there are zero individuals noticed within orientation or attraction regions, thus di $(\mathrm{t}+\mathrm{T})=\mathrm{vi}$.

A bird that turns at a rate of $\gamma$ degrees per second $(\%)$ can turn by an angle equal to $\gamma \mathrm{T}$ degrees each $\mathrm{T}$ sec at most. Then in the case when the angle that lies between $\operatorname{di}(\mathrm{t}+\mathrm{T})$ and vi( $\mathrm{t})$ is lower than $\gamma \mathrm{T}$, at that time the ith bird would be aligned with the calculated vector of the direction, vi $(\mathrm{t}+\mathrm{T})=\operatorname{di}(\mathrm{t}+\mathrm{T})$; or else it would turn only $\gamma \mathrm{T}$ degrees in its direction. After completion the estimation of the next direction vector, the calculation of the next position vector for ith bird is ready to be calculated using the formula: $\mathrm{pi}(\mathrm{t}+\mathrm{T})=\mathrm{vi}(\mathrm{t}+\mathrm{T}) * \mathrm{TU}+\mathrm{pi}(\mathrm{t})$, in this equation U represents bird's speed. In order to investigate and evaluate the combined behavior using the suggested model, consequently, we be able to discover the ultimate results of the fluctuating values that are set to factors which is illustrated in Table 1.

Table 1. The Summary Factors of the Model

\begin{tabular}{lccc}
\hline \multicolumn{1}{c}{ Parameter } & Unit & Symbol & Parameter Range \\
\hline Number of individuals & None & $N$ & $10-100$ \\
Region of repulsion & Units & $r_{r}$ & 1 \\
Region of orientation & Units & $\Delta r_{o}=\left(r_{0}-r_{r}\right)$ & 5 \\
Region of atraction & Units & $\Delta r_{a}=\left(r_{a}-r_{0}\right)$ & 10 \\
Field of perception & Degrees & $\beta$ & 210 \\
Tuming rate & Degrees per second & $\gamma$ & 50 \\
Speed & Units per second & $\mathrm{U}$ & $1-5$ \\
Time step increment & Seconds & $\mathrm{T}$ & 0.1 \\
\hline
\end{tabular}

\section{THE PROPOSED SWARMING MODEL DESIGN AND IMPLEMENTATION}

Firstly, several researchers has been carried out and take on more or less motion direction and constant speed for folks surrounded by resident neighborhood all through emerging a model with a collective motion. In this proposed paper, a genuine biological model was created and developed to simulate flight of bird flocks collective behavior which is based on flocking tendency concept. The computer-generated algorithm of gathering individuals was created wisely and subjected to the alignment, positional repulsion and attractive effect that is depends on the relative posture and direction of these individuals. Group formation and cohesion is achieved as a result of the individual behavior described in this model. The performed simulation manifests characteristic flocking behaviors, exactly like to those seen organisms, even when specific factors are varied. The suggested comprehensive justification of the behavioral using pseudo code program for bird flocks swarming algorithm is as follows: 
Bird _ postures _ initalisation ()

LOOP

Plot_birds ()

Transfer_birds _ to _ next _ postures ()

END LOOP

Bird _ postures _ initalisation () process places a starting locations for all birds. These locations were selected randomly and close to the center of the showing zone. Once the simulation performed, the entire birds take wing towards expected target direction. On the other hand, as the simulations sustained its running state, birds keep an eye and pursued the randomly continuous move target.

Plot _ birds () process would be used to draw a bird motion with single frame, in the existing posture entire birds drawn. The proposed model swarming for birds flock operates optimally with two or even with three dimensions.

Transfer _ birds _ to _ next _ postures () process consist of an algorithm for real birds flocking. Simply, such algorithm work on vector manipulations on birds' postures vectors. Each one of the swarming is work independently from others, consequently for each bird. So, we can calculates bird movements caused by every single one of these three rules that been mentioned and well described previously, resulting three vectors with various velocities. In addition, adding the resulting vectors with the current velocity of the bird, therefore we be able to predict the next velocity of a bird. Accepting that the velocity of a bird is a value were bird travels per time step, then the next bird position can be calculated by simply adding bird velocity due to the three rules to the present position of the bird.

The process mentioned above can be presented by means of pseudo-code as follows:

PROC transfer _ birds _ to _ next _ postures ()

Vector vel1, vel2, vel3, vel4, ...

bird $b$

FOR EACH BIRD b

vel1=rul1(b)

vel2=rul2(b)

vel3=rul3(b)

vel4=rul4(b)

b.vel $=$ b.vel + vel $1+\operatorname{vel} 2+\operatorname{vel} 3+\operatorname{vel} 4+\ldots$

b.pos $=$ b.pos + b.vel

END

END PROC

The three rules uses pseudo-code for the proposed algorithm once a time are as follows:

Rule 1: Bird attempts maintaining a minor separation distance of different birds (counting the obstacles and walls of the surrounded space).

This rule is to ensure that the bird will avoid the collision with another bird within the flock. To apply this rule, a case study autonomous flying taxi will be taken, it will represent one of critical cases is a process of mass parking for these flying vehicles. During this rule, a continuous monitoring of the neighboring birds is performed, and in the case when any neighboring bird is within a specified lower separation distance, for example 50 measuring units, then transfer the bird away from that neighboring bird. This can be accomplished via the subtraction of the displacement of each neighboring bird from an arbitrary vector, say sp.

The vector sp calculated here is initialized at 0 and it added to the bird present position to transfer the bird far from the birds flying beside it. And in pseudo code:

PROC rull(bird bI)

Vector $\mathrm{sp}=0$;

FOR EACH BIRD b

IF $b$ != bI THEN

IF |b.pos - bI.pos $\mid<50$ THEN

$\mathrm{sp}=\mathrm{sp}-$ (b.pos - bI.pos)

END IF

END IF

END

RETURN sp

END PROC 
In the case of two birds are side by side, the previous rule is suitable to apply for both. Therefore both of them will start gradually turned away from the other and the same procedure will take place in next time step that's of course if they are still near each other. They will continue steered more away from each other until they accede the lower separation distance between them. For this reason this will take a smooth or gradual acceleration. Keeping in mind, that is more realistic to ensure smooth movements during the simulation.

Rule 2: Birds attempt to fly in the direction of the center of neighboring birds. The identified neighboring birds' center is simply an average positions of the entire neighboring birds. On behalf of bird bI $(1<=\mathrm{I}<=\mathrm{N})$, the obvious center acI is presented as follows:

$\mathrm{acI}=(\mathrm{b} 1 \cdot \mathrm{pos}+\mathrm{b} 2 \cdot \mathrm{pos}+\ldots+\mathrm{bI}-1 \cdot \mathrm{pos}+\mathrm{bI}+1 \cdot \operatorname{pos}+$

$\ldots+$ bN.pos $) /(\mathrm{N}-1)$

Once a calculation made to determine the apparent center, it is essential to transfer a particular bird in the direction of the intended center. We can determined this process for transferring the bird 0.01 apart from the center via the following:

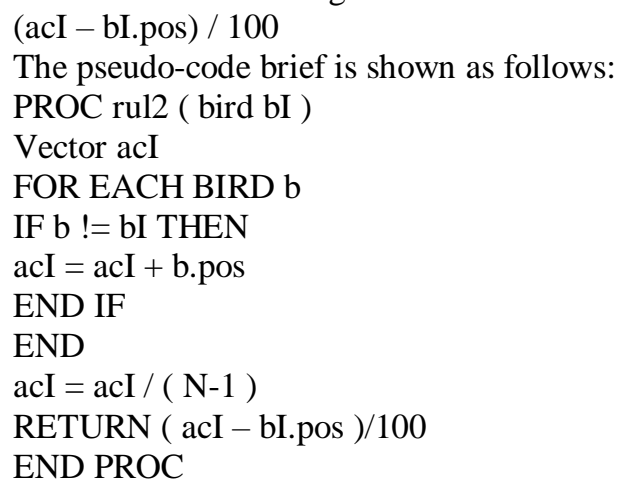

Hence, the first velocity calculation of vector (v1) for a specific bird was successfully achieved.

Rule 3: Bird attempt to sustain same velocity as that of the near birds.

This may be similar to Rule 2, but in its place of taking the average of the neighboring birds' positions, the averaged velocities of the neighboring birds 'apparent velocity' (avI) can be determined, plus increase only a minor fraction, roughly one sixth of bird's current speed. As a result, a gradual boost velocity delivered for the 'apparent velocity'.

PROC rul3(bird bI)

Vector avI

FOR EACH BIRD b

IF $b$ != bI THEN

$\mathrm{avI}=\mathrm{avI}+\mathrm{b} . \mathrm{vel}$

END IF

END

$\operatorname{avI}=\operatorname{avI} /(\mathrm{N}-1)$

RETURN (avI - bI.vel) / 6

END PROC

The birds flocking rules described above are appropriately demonstrate the complicated aggregation behavior seen in bird flock. These are the essential rules that are required in order to mimic the leaderless, Spatial flocking behavior. This requires the provision of communication and control devices (for this particular case), in which represents a process of imitating the role of the commander to maintain the system and regulate the entry process to the main gate, by add a WSN notes (or motes) with microcontroller and Zigbee X3 communication systems or middle-distance communication and remote control. Each of which will act as the leader of the group (based on the first in, will take the leadership) and the leadership then transferred to the last out bird from the remaining swarm. However to acquire a more realistic model for birds flock behavior, other features should be embedded, some of the huge features seen in birds flock behavior are introduced in the following sections. These rules could be added to the transfer_birds_to_next_postures() procedure. 


\subsection{Action of Wind Currents}

PROC wind_currents(Bird b)

Vector current

RETURN current

END PROC

This procedure returns the value of wind currents which is the same for every bird within the flock; therefore the overall birds flock would have the identical current pushing effect.

\subsection{Attraction to a Specific Place or Target}

PROC attract_to_plc(Bird b)

Vector plc

RETURN (plc - b.pos) / 100

END PROC

This procedure transfers the bird 0.01 of its way in the direction of the target at each time step. This will ensure a smooth reach to the target mainly for distant targets.

\subsection{Limitation of Bird Speed}

Limiting the magnitude of the bird speed gives the model a realistic sense, in this manner the birds wouldn't fly in excessive speed. If this limitation is not performed, then the speed of the bird may fluctuate, and there will be a good chance for the birds to fly in a very high speed. Actually the real bird can't fly illogically very fast. For a limiting speed to vmx the following procedure may be used:

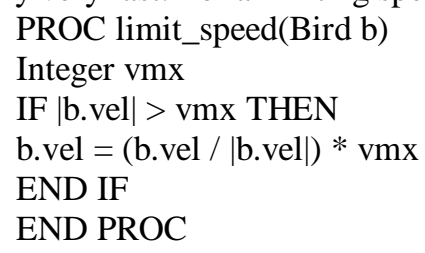

In this procedure, a unit vector of the bird speed was calculated by dividing b.vel by its magnitude, next the specific unit vector is multiplied via vmx. The velocity vector of the bird is now has an equivalent orientation in compare with the original velocity, however the magnitude is equal to vmx. The well-explained process above have to be named inside transfer _ birds _ to _ next - postures () procedure, and it revealed below:

$$
\begin{aligned}
& \text { b.vel }=\text { b.vel }+ \text { vel } 1+\text { vel } 2+\text { vel } 3+\text { vel } 4+\ldots \\
& \text { limit_speed(b) } \\
& \text { b.pos = b.pos + b.vel }
\end{aligned}
$$

\subsection{Limitation the Region of Movement}

To enforce the birds flock to fly within a specific region, as an example on a screen of the computer, instead of using an unrealistic method by using specific boundaries which causes some sort of bouncy from a hidden barriers, one can use another rule which pushes the birds to fly inside rough confines. In this manner the birds could fly out of these borders, but they return slowly, with smooth movements.

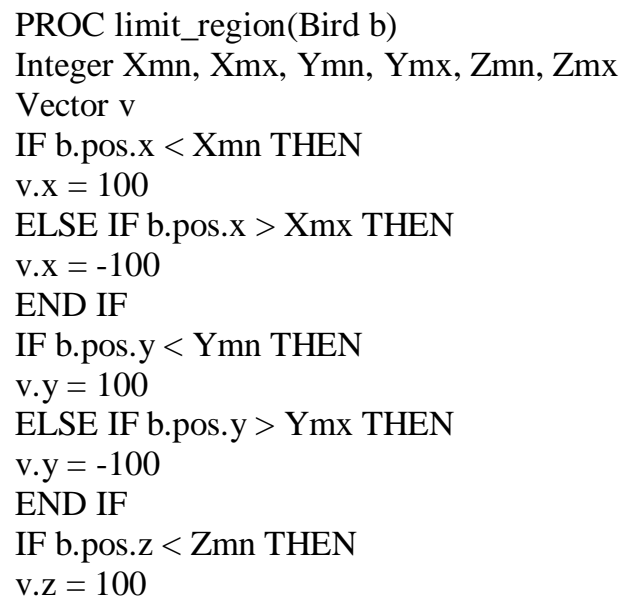




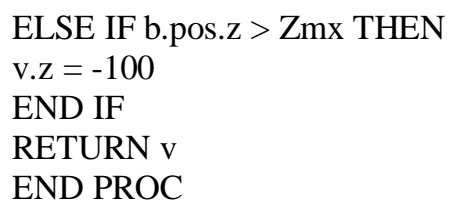

The value 100 used in this procedure is any roughly amount to push the bird to fly in a specific space.

\subsection{Flock Scattering}

Basically, it can scatter birds flock or else interrupt the coherence situation, taking example a flock is surprised using a raucous clatter. Then this may leads to invalidate a portion of the effect of the bird rules. For example, negating first rule (collision avoidance) would merely push the birds to collide each other.

Whereas negating rule no. 2 (moving on the way to neighbor's center) impulse a bird to fly far away from the center of neighbors. Furthermore, negating rule no. 3 (keeping the velocity similar to that of nearby birds) may result in an oscillation and chaotic movements. By using random multipliers with each rule, one can varies the effect of the rules during the simulation time. The multipliers could be putted within the transfer_birds_to_next_postures() procedure, as can be seen in the following code:

PROC transfer_birds_to_next_postures()

Vector vel1, vel2, vel3, vel4, ...

Integer I1, I2, I3, I4, ..

Bird b

FOR EACH BIRD b

vel1 $=\mathrm{I} 1 * \operatorname{rul} 1(\mathrm{~b})$

$\operatorname{vel} 2=\mathrm{I} 2 * \operatorname{rul} 2(\mathrm{~b})$

vel3 $=\mathrm{I} 3 * \operatorname{rul} 3(\mathrm{~b})$

vel4 $=\mathrm{I} 4 * \operatorname{rul} 4(\mathrm{~b})$

$\cdots$

b.vel = b.vel + vel $1+\operatorname{vel} 2+\operatorname{vel} 3+\operatorname{vel} 4+\ldots$

$\ldots$

b.pos $=$ b.pos + b.vel

END

END PROC

I1 need to be negative and there is no need to change vel2, vel3, vel4, . . by Is.

\subsection{Tendency to be Far from a Specified Place}

When the birds flock is required to fly far from a specific location or a Predatory animal, then in this case each bird needs to fly far from that place. The computations needed here are the same as that of flying in the direction of that place, which is performed previously as attract_to_place; in this case, the only difference is to multiply the velocity vector by a negative multiplier:

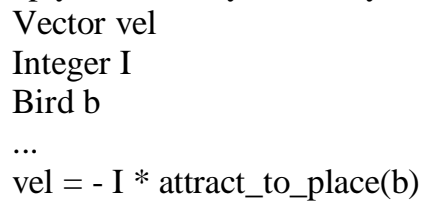

\section{PROPOSED COMMUNICATION SCHEM}

The communication system proposed in this paper does not constitute the basic system of communication between the main station [20-21], the control tower and the flying plane, but an additional automatic system in which activated when the aircraft lays under conditions of the swarm system (in both takeoff and landing cases). Figures $5 \mathrm{a}$ and $5 \mathrm{~b}$ describe the mechanism where this backup system to be use, it is limited to the application of rules (rule1 and rule3) were mentioned in section II before. It means, maintaining small distance intervals when the case is mass departures (eg in the case of flying taxi) or in the case of mass take off (in the parking of similar applications). 


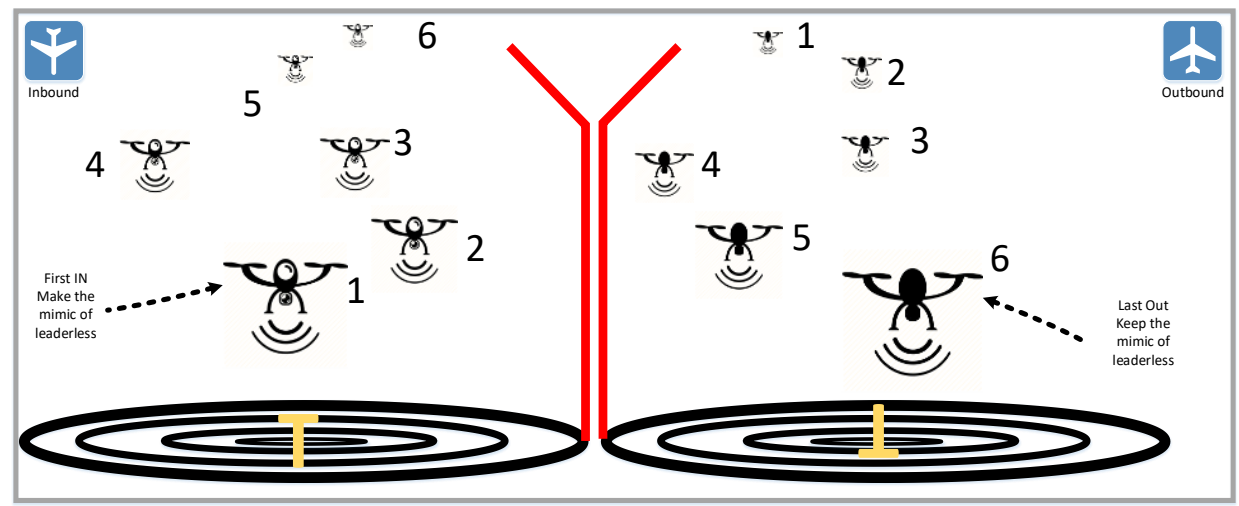

Figure 5. (a) The mass landing, (b) the mass takeoff (parking)

Figure 6 shows the mechanism of leadership transmission (when entering and exiting the area referred to in Figures 3 and 4. This mechanism depends on changing the topology of the communication network according to the status and priority of entry of the bird into the common area (entry and exit gates).

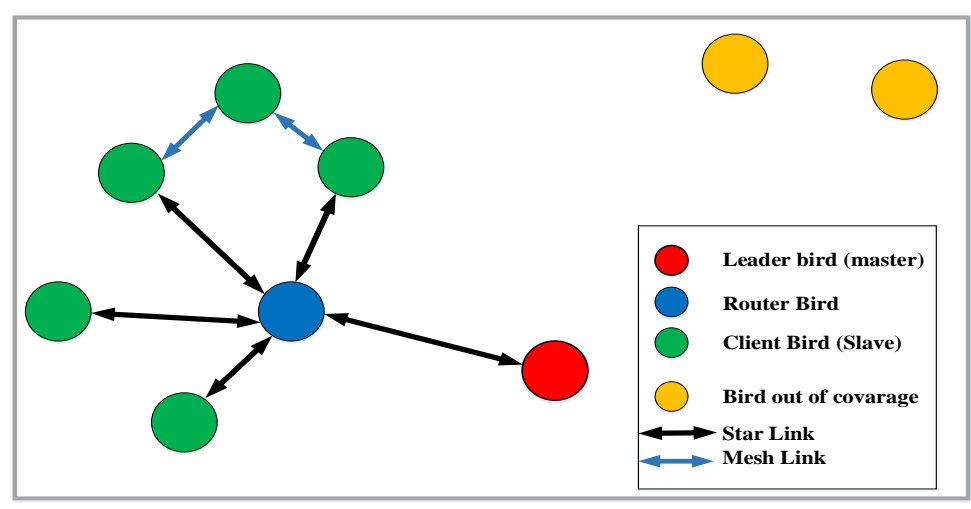

Figure 6. the multiuse of network topologies.

Figure 7 shows the proposed dataflow diagram to solve this issue. This system should preferably be isolated from the basic communication system of the aircraft, that used to communicate with the central network in the control towers.

These systems are equipped with a microcontroller with a Zigbee communication system (such as . This system takes the lead in driving by simply applying the operating conditions (getting closer to other flying objects) and the distance between each other and the starting base station

This data is read and calculates the distances required for the application of (rules 1, 2, and 3) according to the coordinates from the aircraft GPS system, With the aid of these data, the leader pilot will send the instructions to the other members of swarm (to prevent collisions or critical close among the members), which lead to the organize of the process of entry and exit during the mass takeoff and landing operations.

\section{SIMULATION RESULTS AND DISCUSSION}

The suggested bird flocking algorithm was simulated effectively in MATLAB. Moreover, the genuine biological model implementation was positively completed in 3-D distribution space. The simulation has revealed supposing the following; a bird of length $15 \mathrm{~cm}$, number of birds in flock is 30, average linear velocity of bird is $4 \mathrm{~m} / \mathrm{s}$ and average angular velocity of bird is $100 \%$. In additon, all birds fly in the direction of the predictable target (red hollow circle) as we pointed out previously. The results of the proposed simulation program is shows a very promising and wished-for movements (self-orgnised) and it illustrated in Figuers 5, 6 and 7. 


$\begin{array}{|ll|} & \begin{array}{l}\text { disconnected Nodes } \\ \text { (birds) wich lies far }\end{array} \\ \text { away from base } \\ \text { station }\end{array}$

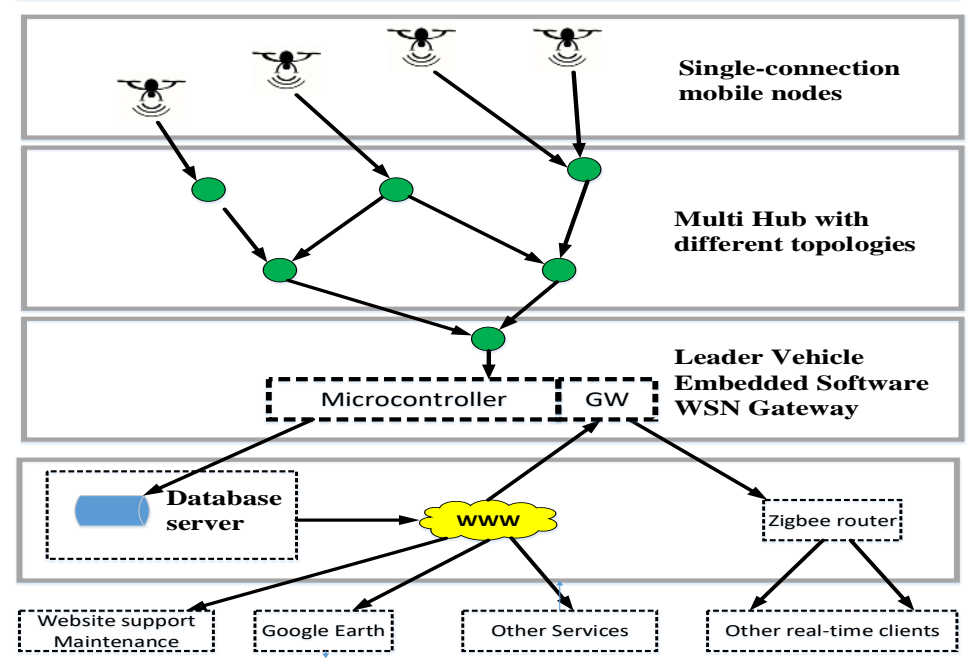

Figure 7. The proposed dataflow diagram.
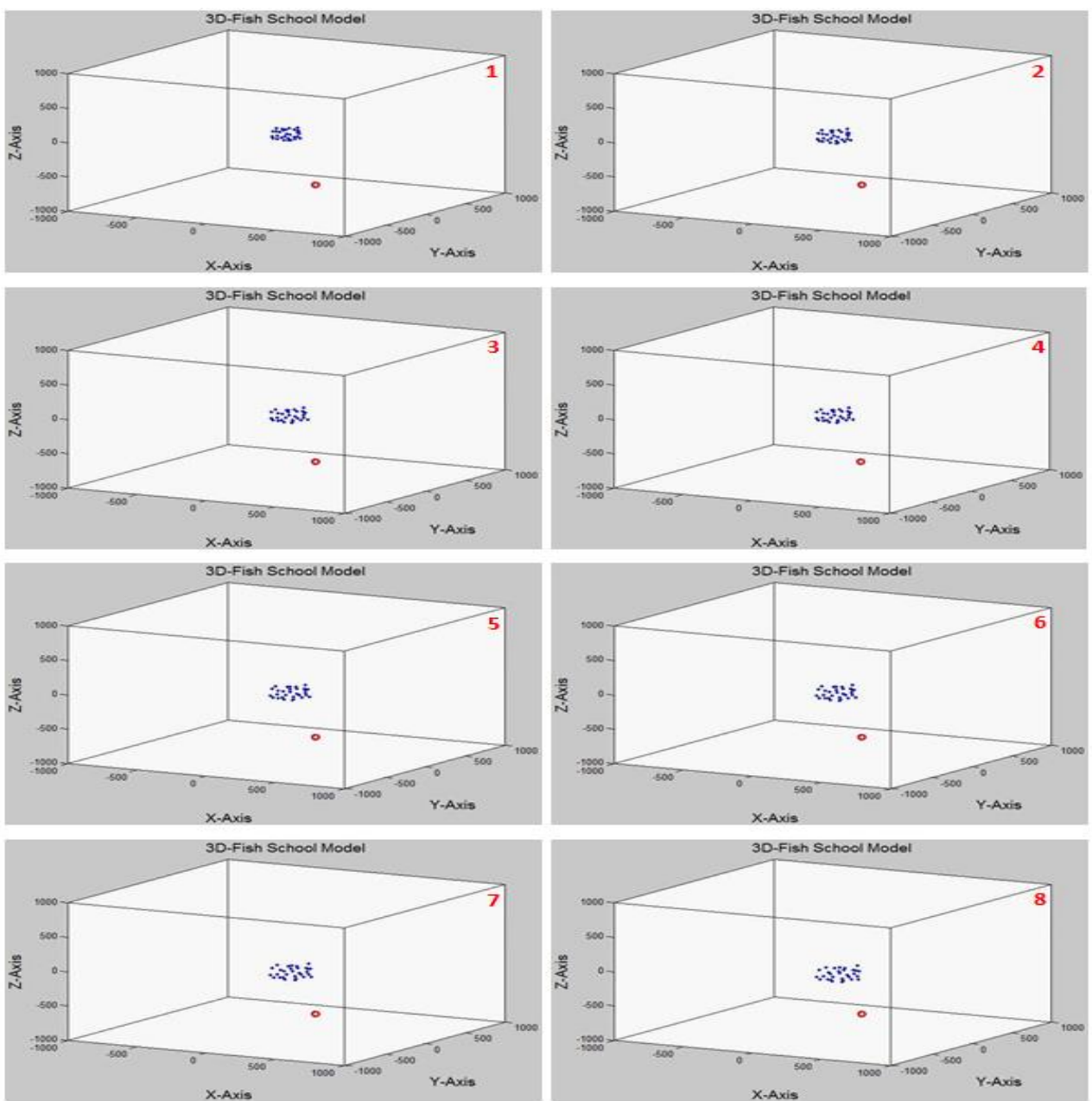

Figure 5. Simulation results of birds flock swarming algorithm in three dimensions (Part 1) 

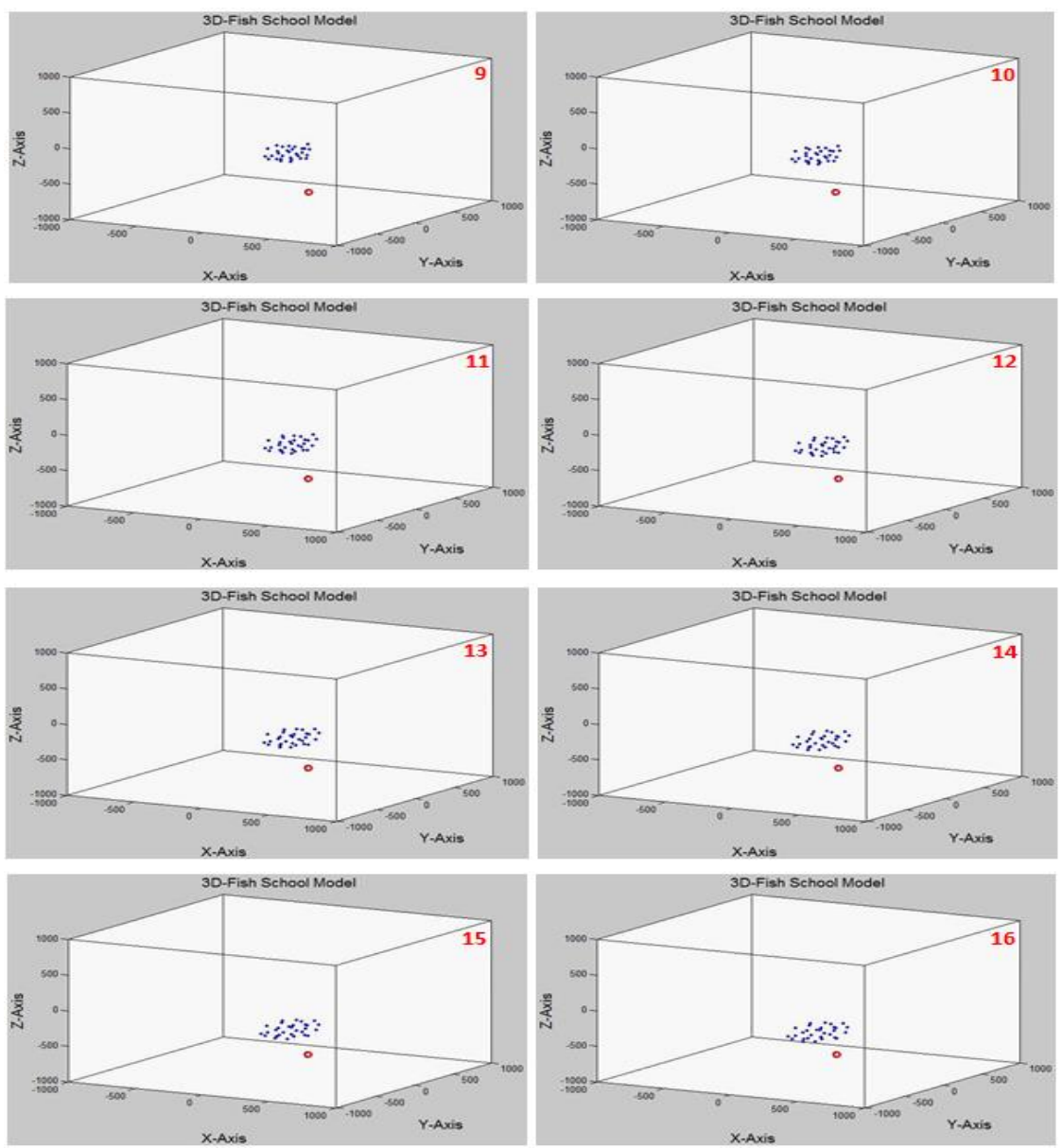

Figure 6. Simulation results of birds flock swarming algorithm in three dimensions (Part 2)
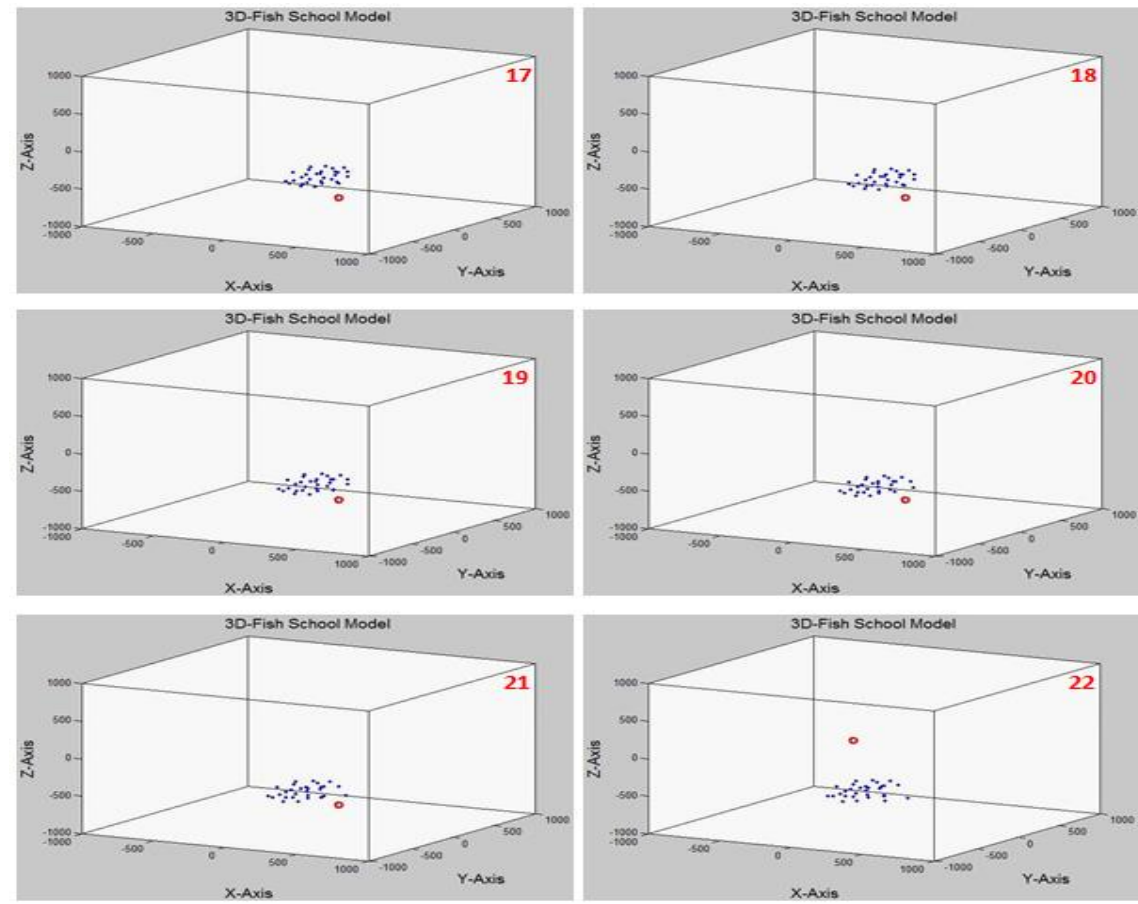

Figure 7. Simulation results of birds flock swarming algorithm in three dimensions (Part 3) 


\section{CONCLUSION}

In conclusion, this paper presents an optimal self-organised model concerning birds flock. The results approves that a 3-d space of a group formation which is ruled via a set of simple spatial rules reflect to a large degree the actual behavior of the flocking birds. Also, it was proved that the biological systems which are based on a collective behavior of their individuals represent an important source in the development of the controlling algorithms of multi mobile robots systems. Furthermore, it was seen that the controlling rules could be extracted experimentally by the continuous monitoring of these biological systems. To sum of all, an independent communication and control systems has been proposed successfully. Similarly, the results shows an optimal self-controlled movements, which can be used to optimize aircraft pilot systems in swarm robotic for flying auto-controlled vehicles and in case of collective takeoff and collective landing swarm-robots.

\section{REFERENCES}

[1] A. Okubo, "Dynamical aspects of animal grouping: swarms, schools, flocks, and herds", Adv. Biophysics., vol.22, pp.1-94, 1986.

[2] M. Rashid, "Design Model for Wireless Multi-Mobile Robot System Based on Modeling of Collective Motion of Artemia Population", Ph.D. thesis, University of Basrah, Basrah, Iraq, 2011.

[3] D. Mark, "Game Programming Gems", Charles River Media, pp. 401-415, 2007.

[4] Poorva Agrawal, Himanshu Agrawal, Vidyasagar Potdar, " A novel bio- inspired algorithm for hunting in multi robot scenario", Indonesian Journal of Electrical Engineering and Computer Science (IJEECS), Vol. 15, No. 3, , pp. 1553 1563, September 2019.

[5] [Reynolds, "Flocks, birds, and schools: a distributed behavioral model", Computer Graphics, vol.21, pp. 25-34, 1987.

[6] T. Vicsek, A. Czirok, E. Ben-Jacob, I. Cohen, and O. Shochet, "Novel type of phase transitions in a system of self driven particles", Physical Review Letters, vol.75, pp.1226-1229, 1995.

[7] A. Jadbabaie, J. Lin, and A.S. Morse, "Coordination of groups of mobile autonomous agents using nearest neighbor rules", IEEE Trans. Aut. Control, vol. 48, pp. 988-1001, 2003.

[8] Tanner H G, Jadbabaie A, Pappas GJ., "Stable flocking of mobile agents, Part I: Fixed Topology”, Proc. of 42nd IEEE Conference on Decision and Control, pp.2010-2015, 2003.

[9] Tanner H G, Jadbabaie A, Pappas GJ., “Stable flocking of mobile agents, Part II: dynamic topology”, Proc. of 42nd IEEE Conference on Decision and Control, pp.2016 - 2021, 2003.

[10] R. Olfati-Saber and R. M. Murray, "Flocking with obstacle avoidance: cooperation with limited communication in mobile networks", in Proc. 42nd IEEE Conf. Decision and Control, vol. 2, pp.2022-2028, 2003.

[11] R. Olfati-Saber, "Flocking for multi-agent dynamic systems: algorithms and theory", IEEE Trans. Automatic Control, vol.51, pp.401-420, 2006.

[12] J. A. Fax and R. M. Murray, "Information flow and cooperative control of vehicle formations", IEEE Trans. Auto. Control, vol. 49, pp.1465-1476, 2004.

[13] Ade Silvia Handayani, Siti Nurmaini, Irsyadi Yani, Nyayu Latifah Husni, " Analysis on swarm robot coordination using fuzzy logic", Indonesian Journal of Electrical Engineering and Computer Science (IJEECS), Vol. 13, No. 1, pp. 48-57, January 2019.

[14] B. Lei, W. Li, and F. Zhang, "Flocking Algorithm for Multi-Robots Formation Control With a Target Steering Agent”, 2008 IEEE International Conference on Systems, Man and Cybernetics (SMC 2008).

[15] R. Carelli, and E. Freire, "Corridor navigation and wall-following stable control for sonar-based mobile robots", Elsevier, Robotics and Autonomous Systems, Vol. 45, pp. 235-247, 2003.

[16] Siegwart, R. and Nourbakhsh, I. R., Introduction to Autonomous Mobile Robots, MIT Press, Cambridge, UK, 2004.

[17] Siti Nurmaini, Bambang Tutuko, "Intelligent Robotics Navigation System: Problems, Methods, and Algorithm International Journal of Electrical and Computer Engineering (IJECE), Vol. 7, No. 6, pp. 3711-3726, December 2017.

[18] Antonio L.Alfeo, Mario G.C.A.Cimino,, Nicoletta De Francesco, Massimiliano Lega and Gigliola Vaglini, " Design and simulation of the emergent behavior of small drones swarming for distributed target localization", Journal of Computational Science, Volume 29, , Pages 19-33, November 2018.

[19] Z. ALLAWI, "Fuzzy Logic Control Scheme for Navigation of Multiple Mobile Robots", Ph.D. thesis, University of Basrah, Basrah, Iraq, 2014.

[20] Jin-Yong, Lin, et al. "Investigation of Network Communication Protocol on Autonomous Aerocraft Flying Formation." 2015 Fifth International Conference on Instrumentation and Measurement, Computer, Communication and Control (IMCCC). IEEE, 2015.

[21] Cantas, Mustafa Ridvan, et al. "Cooperative Adaptive Cruise Control Design and Implementation", No. 2019-01-0496. SAE Technical Paper, 2019. 